\title{
Definição de características críticas na implementação de serviços customizados em massa
}

\author{
Gabriel Vidor ${ }^{\mathrm{a} *}$, Janine Fleith de Medeiros ${ }^{\mathrm{b}}$, Flávio Sanson Fogliatto ${ }^{\mathrm{c}}$ \\ a*gvidor@producao.ufrgs.br, UFRGS, Brasil \\ bjanine@upf.br, UFP, Brasil \\ cffogliatto@producao.ufrgs.br, UFRGS, Brasil
}

\begin{abstract}
Resumo
O objetivo deste trabalho é definir quais características de customização em massa (CM) presentes em sistemas de manufatura podem ser priorizadas no projeto de serviços customizados em massa. Propõe-se uma classificação de importância de características de customização a serem priorizadas na implementação de serviços customizados, tendo-se como critérios os habilitadores de $\mathrm{CM}$ e os habilitadores de serviços combinados através de uma matriz de relacionamentos característica do QFD (desdobramento da função qualidade) e corrigidos, a partir da ponderação dos gestores do serviço, usando-se um procedimento de QFD reverso. No final do estudo foi possível determinar as características prioritárias do serviço customizado em massa. Dois casos de estudo foram realizados para exemplificar a operacionalização da metodologia, um em uma distribuidora de energia elétrica e outro em uma universidade privada.
\end{abstract}

Palavras-chave

Customização em massa. Serviços. QFD.

\section{Introdução}

A customização em massa (CM) apesar de apresentar soluções potenciais para a área de manufatura, com estudos consolidados nas áreas de cadeia de suprimentos (ABDELKAFI et al., 2010), análise de mercado e planejamento da estrutura de produtos (SPRING; ARAUJO, 2009) e planejamento e controle da produção (ZHANG; CHEN, 2006; TSENG; RADKE, 2010; LEE; DAl, 2010), ainda é alvo de questionamentos sobre sua real aplicabilidade na área de serviços (McCARTHY; PITT; BERTHON, 2010).

Essas questões decorrem, em parte, da subjetividade do termo customização em massa, além das peculiaridades da gestão em serviços, considerando suas características distintas (WITELL et al., 2011). Sabe-se que o conceito CM tem sido aplicado em diversos contextos, tanto na prestação de serviços quanto na manufatura. Todavia, a aplicabilidade do conceito faz com que as apropriações em relação ao termo customização em massa tornem-se diversas e, ainda que complementares, apresentem algumas diferenças em relação ao conceito original. Por exemplo, termos como estratégia de manufatura, prestação de serviço, modificações na cadeia de suprimento e planejamento de marketing são comumente encontrados na literatura para definir a CM. Segundo Davis (1987), customização em massa originalmente refere-se a uma estratégia de negócio para proporcionar aos clientes o que eles querem, no tempo e na forma desejados. Conceitualmente, a CM pode ser entendida como uma estratégia de negócios que diferencia as organizações em ambientes de alta competitividade e torna mais qualificada a segmentação de mercado (HELO et al., 2010).

Entende-se, nesse trabalho, a CM como uma estratégia que permite ofertar produtos e serviços com grau de variedade que atenda às necessidades do cliente e apresente custos similares aos dos itens produzidos em massa. A oferta de variedade garante que a empresa seja capaz de atender aos requisitos de personalização, o primeiro elemento do binômio customização/massa. Os custos similares aos da produção em massa garantem que a empresa atenda 
ao segundo elemento: o da produção a um custo que racionalize a operação de manufatura e a prestação do serviço. Essa definição serve para nortear as discussões do presente trabalho.

Apesar da evolução da CM na indústria, a solução conceitual para a área de serviços ainda é incipiente (PETERS; SAIDIN, 2000). lsso porque o setor de serviços apresenta altos graus de intangibilidade, perecibilidade, inseparabilidade e variabilidade (ORDANINI et al., 2011), conceitos nem sempre presentes na manufatura, onde os estudos de CM tiveram sua origem. Os serviços customizados caracterizam-se essencialmente pela: ( $i)$ heterogeneidade de demandas de mercado, (ii) velocidade e variedade exigidas pelos clientes nesse tipo de segmento, e (iii) competição com empresas do mesmo segmento (CAO et al., 2006). Tais características, combinadas aos diferentes níveis em que a customização pode ocorrer (PAN; HOLLAND, 2006; DA SILVEIRA et al., 2001), acaba dificultando a vinculação entre serviços e CM.

Observa-se na literatura que a área de serviços customizados apropria-se das características de manufatura customizada, não havendo uma classificação das características de customização sob a perspectiva de serviços. Uma abordagem para obter características de serviços customizados consiste na utilização das características de manufatura e verificação daquelas possíveis de utilização na caracterização de "serviços customizados em massa" (McCARTHY; PITT; BERTHON, 2010).

Nesse sentido, o objetivo deste trabalho é desenvolver uma sistemática para definir quais características de CM para manufatura devem ser priorizadas no projeto de serviços customizados em massa. Para tanto, propõe-se uma ponderação da importância de características de customização com vistas à implementação de serviços customizados, tendo-se como critérios os habilitadores de customização em massa e os habilitadores de serviços, combinados através de uma matriz de relacionamentos característica do QFD (Quality Function Deployment ou desdobramento da função qualidade) e corrigidos, a partir da incorporação do julgamento dos gestores do serviço em estudo, usando-se o procedimento reverso do QFD proposto por Fogliatto e Da Silveira (2008).

0 presente artigo traz duas contribuições importantes para a área de gestão de serviços: primeiro, ao mapear características e habilitadores da CM, típicos das operações de manufatura, com relação a dimensões que caracterizam uma prestação de serviços; segundo, ao customizar a análise a um contexto específico de projeto de serviço, através das operações reversas realizadas nas matrizes de relacionamentos. No referencial teórico apresentado na seção 2 não foi possível encontrar estudos similares.
0 restante do artigo está organizado em 4 seções. Na seção 2 é apresentado um referencial teórico sobre customização em massa, destacando trabalhos que associam a CM a serviços. $\mathrm{Na}$ seção 3 , de materiais e métodos, apresenta-se a proposta metodológica, a qual é aplicada em dois estudos de caso, descritos na seção 4. Conclusões encerram o artigo na seção 5.

\section{Referencial teórico}

A literatura sobre customização em massa na área de serviços apresenta ênfase no desenvolvimento de modelos voltados à estratégia da empresa, vinculando essa estratégia ao planejamento do serviço customizado. Alguns trabalhos relevantes sobre o assunto são apresentados na sequência.

Cao et al. (2006) desenvolveram um método para a customização do serviço. Nele foi definida uma rotina de customização baseada no processo de venda de passagens aéreas, onde a compra de cada cliente foi analisada e usada para remodelar a estrutura do serviço. Através desse estudo, foram apresentados os impactos estratégicos e de qualidade nas características do serviço customizado, além de um sistema de classificação de solicitações de serviços de clientes para companhias aéreas.

Helms et al. (2008) defendem a customização do serviço através do comércio eletrônico, ainda que não apresentando um método ou modelo de customização para tal. No trabalho, há uma associação entre a utilização da internet e os aspectos intangiveis dos serviços para explicar como integração de sistemas, inovação em produto, mensuração de desempenho, estratégia da organização e economia em custos são importantes para a prestação do serviço. Os autores concluem que a utilização de ferramentas de internet e das informações dos clientes (através de armazenagem em bancos de dados) representam uma vantagem competitiva nos serviços customizados. Um modelo como esse também poderia ser usado para definir a estratégia de um serviço customizado, como proposto no presente artigo. Contudo, no modelo de Helms et al. (2008) não existe qualquer informação que permita ou indique essa aplicação.

Jin, He e Song (2011) discutem a customização de serviços exemplificando-a através da elaboração de um pacote de viagens. Em uma amostra de 220 casos na qual se realizou uma análise discriminante foi possivel ponderar a importância do aumento ou redução da customização associada ao serviço. Um modelo de regressão logística, composto de variáveis comportamentais e psicológicas, permitiu identificar os atributos essenciais e periféricos para a oferta do serviço customizado. Os resultados 
obtidos comprovaram que uma maior customização do serviço pode aumentar a satisfação e lealdade dos clientes. Em contrapartida, verificou-se que a maior customização implica também em maior custo para aquele que a oferta.

Grenci e Watts (2007) propuseram uma estrutura para associar customização nas vendas com a customização da produção. Para os autores, a customização da produção depende da existência de sistemas de informação, modularidade e interconectividade; as vendas customizadas apresentam três classes correspondentes: informação para decisão, agregação e comércio eletrônico. Assim, estabeleceu-se uma relação direta entre os grupos de características de manufatura e de venda, permitindo concluir que as vendas customizadas dependem do segmento de mercado e seu potencial de crescimento, do cliente (sua experiência e comportamento) e da qualidade dos dados coletados do cliente no serviço de venda.

Bask, Tinnilä e Rajahonka (2010) desenvolveram um modelo em que as etapas da cadeia de serviços logísticos são analisadas como fases modulares de um processo, tornando-as customizáveis dentro desse processo. A partir do modelo, as demandas dos clientes são transformadas em processos, os quais são agrupados em módulos de serviços logísticos, o que permite a customização do serviço de entrega. 0 modelo, entretanto, não permite medir a eficiência da customização, sendo indicado apenas na determinação da estratégia a ser adotada na organização.

Tang et al. (2010) propuseram uma estrutura que descreve o processo de decisão de compra do cliente em relação aos produtos e serviços customizados. Essa estrutura é baseada em 6 categorias e 24 sub-categorias que servem para avaliar a customização, seus determinantes e suas consequências. Os resultados indicam que a customização em massa de serviços pode ser organizada em seis etapas, sendo a primeira etapa a principal contribuição do trabalho. Nessa etapa ocorre a criação do conhecimento, estabelecendo uma relação permanente entre clientes e empresa com a busca de informações preliminares para o desenvolvimento do serviço. As demais etapas seguem a lógica tradicional da resolução dos problemas no desenvolvimento do serviço, partindo da fase inicial de identificação do problema até a fase final de fornecimento customizado.

Outros estudos abordam de forma mais superficial a customização de serviços. Jiao, Ma e Tseng (2003) entenderam a customização do serviço como uma fase do processo de prestação do serviço, que pode ser moldada de acordo com as especificações dos clientes no momento em que a prestação do serviço ocorre. Shamsuzzoha, Kyllönen e Helo (2009) mostraram a customização do serviço como uma parte a agregar na cadeia de suprimentos, onde os fornecedores dos insumos usados na prestação de serviço atendem de forma customizada e viabilizam a prestação do serviço da mesma forma. 0'Shaughnessy e 0'Shaughnessy (2009) entenderam a customização do serviço como uma forma de desenvolver a competitividade, captando o cliente de forma definitiva. Para Gottfridsson (2010), a customização do serviço ocorre na medida em que as organizações projetam o serviço, através da utilização de sistemas business-to-consumer (B2C), sendo essa uma parte do processo de criação do serviço. Para Buffington (2011), a customização do serviço é uma forma de segmentação de mercado que deriva do fornecimento em massa de um determinado produto.

Nenhum dos estudos analisados traz uma fase em que o serviço customizado é pensando a partir de características de customização. As características do serviço customizado podem ser entendidas como um grupo de variáveis que devem ser priorizadas no projeto de implementação do serviço customizado, variando de acordo com a perspectiva ou estratégia de uma determinada empresa. No presente artigo pretende-se definir essa sistemática, visto que trata-se de uma etapa que apoia a elaboração dos modelos de serviços customizados descritos anteriormente.

\section{Materiais e métodos}

0 estudo está caracterizado em cinco etapas: (i) identificação das características de customização em massa; (ii) identificação dos habilitadores de customização em massa; (iii) identificação das dimensões de serviço; (iv) aplicação do método QFD e QFD reverso; ( $v$ ) ilustração do método através de dois casos na área de serviços.

Para identificar as características de CM na etapa (i) foi realizada uma revisão de literatura nas bases de dados Emerald, Sage, ScienceDirect, Springer-Verlag e Kluwer. A pesquisa foi executada a partir das palavras chave mass customization; o período para a busca foi restrito de 1990 até 2010. A restrição do período deve-se ao fato da expressão mass customization ter sido utilizada pela primeira vez no ano de 1987 , não havendo publicações correlatas ao assunto em períodos anteriores. A partir da base de 104 artigos obtidos foi realizada uma revisão sistemática para verificar quais poderiam ser utilizados como suporte ao presente estudo. Dessa forma, restaram 83 artigos nos quais foram identificadas 13 características de customização. Para desdobrar claramente as características associadas ao conceito de CM, os resultados da busca bibliográfica foram organizados no Quadro 1. Nesse caso, a descrição das características buscou focar na área de serviços, conservando o conceito original do artigo em que foi obtida. 
Quadro 1. Características de CM identificadas na literatura.

\begin{tabular}{|c|c|c|}
\hline & Características & Referências \\
\hline $\mathrm{C} 1$ & $\begin{array}{l}\text { Estrutura/arquitetura de serviços } \\
\text { organizada em famílias, plataformas, } \\
\text { módulos e componentes. }\end{array}$ & $\begin{array}{l}\text { Tseng e Jiao (1996), Jiao e Tseng (1999, 2004), Muffato (1999), Dahmus, Zugasti e Otto } \\
\text { (2001), Simpson, Maier e Mistree (2001), Gershenson, Prasad e Zhang (2003), Simpson } \\
\text { (2004), Zha, Sriram e Lu (2004), Corbett e Rosen (2004), Jose e Tollenaere (2005), Huang, } \\
\text { Zhang e Liang (2005), Zhang, Tor e Britton (2005), Marion et al. (2006), Simpson et al. } \\
\text { (2006), Thevenot e Simpson (2006), Dai e Scott (2007), Huang, Zhang e Lo (2007), Jiao, } \\
\text { Simpson e Siddique (2007), Alizon et al. (2007), Mun et al. (2007), Williams et al. (2007), } \\
\text { Fixson (2007), Li, Xue e Gu (2008), Ni, Lu e Yarlagadda (2008), Lindquist, Berglund e } \\
\text { Johannesson (2008), Kumar (2008), Kumar, Gatoufi e Reisman (2008) e Salvador, Martin } \\
\text { e Piller (2009) }\end{array}$ \\
\hline $\mathrm{C} 2$ & $\begin{array}{l}\text { Projetos adaptáveis obtidos por } \\
\text { processos customizados. }\end{array}$ & $\begin{array}{l}\text { Tseng e Jiao (1996), Jiao e Tseng (1999, 2004), Duray et al. (2000), Dahmus, Zugasti e } \\
\text { Otto (2001), Duray (2002), Karlsson (2002), Piller (2004), Corbett e Rosen (2004), Zha, } \\
\text { Sriram e Lu (2004), Gershenson (2004), Zhang, Tor e Britton (2005), Jose e Tollenaere } \\
\text { (2005), Pan e Holland (2006), Dai e Scott (2007), Alizon et al. (2007), lsmail et al. (2007), } \\
\text { Apeagyei e Otieno (2007), Williams et al. (2007), Lindquist, Berglund e Johannesson } \\
\text { (2008), Bare e Cox (2008) e Li, Xue e Gu (2008) }\end{array}$ \\
\hline $\mathrm{C} 3$ & $\begin{array}{l}\text { Integração do cliente no processo de } \\
\text { serviços, permitindo total atendimento } \\
\text { de suas necessidades e expectativas. }\end{array}$ & $\begin{array}{l}\text { Duray et al. (2000), Da Silveira et al. (2001), Anderson-Connell, Ulrich e Brannon (2002), } \\
\text { Bardakci e Whitelock (2003, 2004, 2005), Siddique e Boddu (2004), Zha, Sriram e Lu } \\
\text { (2004), Piller, Moeslein e Stotko (2004), Piller (2004), Sigala (2006), Williams et al. (2007), } \\
\text { Wang e Lin (2008), Haug, Ladeby e Edwards (2009), Cho e Fiorito (2009) e Spring e Araujo } \\
\text { (2009) }\end{array}$ \\
\hline $\mathrm{C} 4$ & $\begin{array}{l}\text { Planejamento de marketing e do } \\
\text { marketing do serviço. }\end{array}$ & $\begin{array}{l}\text { Duray et al. (2000), Anderson-Connell, Ulrich e Brannon (2002), Bardakci e Whitelock } \\
\text { (2003), Mun et al. (2007) e Endo e Kincade (2008) }\end{array}$ \\
\hline $\mathrm{C} 5$ & $\begin{array}{l}\text { Sistemas de informação que } \\
\text { funcionam como canais de } \\
\text { comunicação. }\end{array}$ & $\begin{array}{l}\text { Jiao e Tseng (2004), Piller (2004), Siddique e Boddu (2004), Jiao, Simpson e Siddique } \\
\text { (2007), Alizon et al. (2007), Ma, Jiao e Deng (2008), Ni, Lu e Yarlagadda (2008), Lindquist, } \\
\text { Berglund e Johannesson (2008), Fogliatto e Da Silveira (2008) e Feng et al. (2009) }\end{array}$ \\
\hline C6 & $\begin{array}{l}\text { Customização da cadeia de } \\
\text { suprimentos. }\end{array}$ & $\begin{array}{l}\text { Hoek (2001), Salvador, Forza e Rungtusanatham (2002), Yang, Burns e Backhouse (2004), } \\
\text { Pan e Holland (2006), Huang, Zhang e Liang (2005), Huang, Zhang e Lo (2007), Lindquist, } \\
\text { Berglund e Johannesson (2008) e Jitpaiboon, Dangol e Walters (2009) }\end{array}$ \\
\hline $\mathrm{C7}$ & $\begin{array}{l}\text { Gestão de dados, configurações, } \\
\text { etapas da prestação de serviços. }\end{array}$ & Jiao e Tseng (1999), Ni, Lu e Yarlagadda (2008) e Liou, Yen e Tzeng (2010) \\
\hline $\mathrm{C} 8$ & Determinação de custos por atividade. & Piller, Moeslein e Stotko (2004) e Chen e Wang (2007) \\
\hline C9 & $\begin{array}{l}\text { Integração entre planejamento dos } \\
\text { serviços e as metas da organização. }\end{array}$ & $\begin{array}{l}\text { Duray et al. (2000), Duray (2002), Salvador, Forza e Rungtusanatham (2002), Brown e } \\
\text { Bessant (2003), Jiao e Tseng (2004), Du, Jiao e Jiao (2005), Alizon et al. (2007), Lindquist, } \\
\text { Berglund e Johannesson (2008), Jitpaiboon, Dangol e Walters (2009) e Starr (2010) }\end{array}$ \\
\hline $\mathrm{C} 10$ & $\begin{array}{l}\text { Agilidade e velocidade da prestação } \\
\text { de serviços. }\end{array}$ & Da Silveira et al. (2001), Brown e Bessant (2003) e lsmail et al. (2007) \\
\hline $\mathrm{C} 11$ & $\begin{array}{l}\text { Ferramentas (engenharia concorrente, } \\
\text { sistemas híbridos etc.) e estratégias de } \\
\text { produção (produção enxuta, produção } \\
\text { em massa etc.). }\end{array}$ & $\begin{array}{l}\text { Kotha (1995), Muffatto (1999), Hoek (2001), Salvador, Forza e Rungtusanatham (2002), } \\
\text { Karlsson (2002), Piller, Moeslein e Stotko (2004), Simpson (2004), Bardakci e Whitelock } \\
\text { (2005), Jose e Tollenaere (2005), Zhang, Tor e Britton (2005), Zhang e Chen (2006), } \\
\text { Simpson et al. (2006), Mun et al. (2007), Kincade, Regan e Gibson (2007), Apeagyei e } \\
\text { Otieno (2007), Alizon et al. (2007), Jiao, Simpson e Siddique (2007) e Ni, Lu e Yarlagadda } \\
\text { (2008) }\end{array}$ \\
\hline $\mathrm{C} 12$ & $\begin{array}{l}\text { Criação de conhecimento através de } \\
\text { retroalimentação de informações. }\end{array}$ & Kotha (1995) e Zha, Sriram e Lu (2004) \\
\hline $\mathrm{C} 13$ & $\begin{array}{l}\text { Técnicas de repetição, padronização, } \\
\text { prototipagem e personalização. }\end{array}$ & $\begin{array}{l}\text { Tseng e Jiao (1996), Bardakci e Whitelock (2004), Piller (2004, 2008), Bare e Cox (2008) e } \\
\text { Kumar (2008) }\end{array}$ \\
\hline
\end{tabular}

As características mais referenciadas nos estudos estão associadas à definição da estrutura de serviço (C1), à utilização de projetos e processos adaptáveis (C2), aos tipos de ferramentas e estratégias (C11) e ao atendimento das necessidades de clientes (C3), as quais apareceram, respectivamente, em 43,5\%, 32\%, $29 \%$ e $23 \%$ das publicações.

Na etapa 2, os habilitadores de customização foram obtidos através do estudo de Fogliatto, Da Silveira e Borenstein (2012). A fim de facilitar o entendimento desses habilitadores, eles estão organizados em quatro classes: (i) metodologias, (ii) processos, (iii) tecnologias de manufatura e (iv) tecnologias de informação.
0 habilitador metodologias refere-se ao binômio produzir produtos confiáveis com agilidade (i.e., atendendo as restrições de tempo) e gerar requisitos de customização acessíveis ao uso de princípios enxutos, maximizando a integração e minimizando a perda (FOGLIATTO; DA SILVEIRA; BORENSTEIN, 2012). Nesse sentido, a utilização de princípios e conceitos associados a estratégias enxutas e ágeis são relevantes para implementar a CM.

0 habilitador processos é subdividido em cinco itens. 0 primeiro, denominado elicitação de ordem, associa a busca de informações de clientes através de ferramentas estruturadas de coleta e interpretação 
de dados, para definir a configuração do produto. 0 segundo, postponement, indica o tempo e a forma do atraso na personalização do produto em seu processo produtivo: o tempo indica o atraso associado à chegada do pedido e entrega para o cliente; a forma indica como diferenciações são incorporadas ao produto. Plataforma de produtos é o terceiro item. Conforme Fogliatto, Da Silveira e Borenstein (2012), a plataforma de produtos é uma base comum que consiste de rotinas, atividades e objetos compartilhados que permanecem constantes à medida que novos produtos são gerados. A literatura detalha formas de elaboração de plataformas, cada qual voltada a um determinado propósito. 0 item manufatura permite entender como deve ser o planejamento e controle da produção para itens customizados, englobando ferramentas, máquinas, setups e tempos de ciclo (JIA0; SIMPSON; SIDDIQUE, 2007). Por fim, o item cadeia de suprimentos reflete a coexistência entre fazer sob encomenda e fazer para estoque, explicando como as etapas de geração do produto devem ser conduzidas até a obtenção de itens customizados.

0 habilitador tecnologias de manufatura foca na elaboração do produto (NIELSEN; COX, 2008). Nesse caso, ferramentas como CAD (Computer-Aided Design), FMS (Flexible Manufacturing System), CIM (Computer Integrated Manufacturing) e CNC (Computer Numerical Control) acabam preteridas quando comparadas a técnicas de prototipagem; por exemplo, a utilização de escaneamento a laser integrado a sistemas CAD para a geração do protótipo inicial ao invés da elaboração do protótipo virtual através do CAD.

Por fim, o habilitador tecnologias de informação refere-se particularmente à integração do fluxo de informação internamente na organização e à necessidade de as empresas agregarem aos produtos informações provenientes dos clientes (FOGLIATTO; DA SILVEIRA; BORENSTEIN, 2012). O papel da tecnologia da informação para CM está em incluir o cliente na configuração do produto, na especificação do produto e mesmo na fase de projeto do produto (DIETRICH; KIRN; SUGUMARAM, 2007). A maior contribuição das tecnologias de informação está em aumentar a satisfação do cliente e seu conhecimento em relação às suas preferências (DEAN; TU; XUE, 2009). Simultaneamente, as tecnologias de informação apoiam as opções do cliente quanto às suas compras e às decisões da empresa quanto a preço, projeto, planejamento da produção e gestão da cadeia de suprimentos.

$\mathrm{Na}$ etapa 3 foram elencadas as dimensões de serviços. Verificou-se que segundo Zeithaml (1981), Witell et al. (2011) e Ordanini et al. (2011), serviços são compostos por quatro dimensões: (i) intangibilidade, (ii) perecibilidade, (iii) variabilidade, e (iv) inseparabilidade. A intangibilidade corresponde ao fato de que em serviços o processo é o produto (KOTLER; KELLER, 2006). Por isso, os consumidores de serviços buscam por evidências da qualidade do serviço, tais como instalações, pessoas, equipamentos, material de divulgação e preço (BOOMS; BITNER, 1981; LEVITT, 1991). Perecibilidade diz respeito à incapacidade de estocar serviços. Assim, os gestores de organizações prestadoras de serviços devem promover ações que busquem proporcionar um equilíbrio entre oferta e demanda, tais como preços diferenciados, ações promocionais, serviços complementares e mecanismos de reserva (FITZSIMMONS; FITZSIMMONS, 2010). Variabilidade decorre da dependência do resultado da prestação do serviço nas pessoas. Dessa forma, as organizações que atuam no setor devem investir em processos de contratação e treinamento compatíveis com o posicionamento da empresa, padronizar o processo de execução do serviço e monitorar a satisfação dos clientes (ZAHAJ; GRIFFIN, 2002). Por fim, a inseparabilidade trata da simultaneidade entre prestador e consumidor, quase sempre necessária.

Na quarta etapa, através da análise de especialistas definiu-se a intensidade de relacionamento entre os habilitadores e as características da CM, apresentada nas posições de cruzamento entre as linhas e colunas da Tabela 1.

Na sequência, os especialistas foram solicitados a identificar a intensidade dos relacionamentos entre habilitadores da CM e dimensões que caracterizam os serviços. Os resultados, correspondentes à média da opinião dos especialistas, são apresentados na Tabela 2.

0 grupo de especialistas do qual foram obtidos os resultados nas Tabelas 1 e 2 caracteriza-se por pessoas que trabalham cotidianamente com pesquisa na área de serviços e na área de CM. 0 grupo foi formado por três doutores na área de Engenharia de Produção, três na área de Administração de Empresas e dois doutorandos em Engenharia de Produção. A Tabela 3 caracteriza o grupo de especialistas.

$\mathrm{Na}$ quinta etapa, dois casos foram analisados para ilustrar como empresas de serviços podem organizar a customização do serviço prestado. 0 primeiro caso é o de uma fornecedora de energia elétrica e o segundo, de uma universidade privada. 0 objetivo dos estudos foi identificar habilitadores e características da CM a serem priorizadas na obtenção de serviços customizados, usando como base as relações estabelecidas nas Tabelas 1 e 2 . Para tanto, utilizou-se a análise do QFD tradicional e do QFD reverso, propostas por Fogliatto e Da Silveira (2008). Ambas as análises são apresentadas na sequência. 
Tabela 1. Relações entre características e habilitadores de customização.

\begin{tabular}{|c|c|c|c|c|c|c|c|c|}
\hline \multirow{3}{*}{$\begin{array}{l}\text { Características } \\
\text { de customização } \\
\text { em massa }\end{array}$} & \multicolumn{8}{|c|}{ Habilitadores de customização } \\
\hline & \multirow[b]{2}{*}{ Metodologia } & \multicolumn{5}{|c|}{ Processos } & \multirow[b]{2}{*}{ Sl e Tl } & \multirow{2}{*}{$\begin{array}{c}\text { Tecnologias } \\
\text { de } \\
\text { manufatura }\end{array}$} \\
\hline & & $\begin{array}{c}\text { Elicitação de } \\
\text { ordem }\end{array}$ & Postponement & $\begin{array}{l}\text { Plataforma } \\
\text { de produtos }\end{array}$ & Manufatura & $\begin{array}{c}\text { Cadeia de } \\
\text { suprimentos }\end{array}$ & & \\
\hline $\mathrm{C} 1$ & 5,17 & 7,67 & 6,50 & 8,00 & 7,00 & 6,50 & 6,83 & 6,67 \\
\hline $\mathrm{C} 2$ & 7,00 & 6,67 & 7,00 & 7,83 & 7,17 & 7,33 & 6,83 & 6,83 \\
\hline $\mathrm{C} 3$ & 5,33 & 8,67 & 6,50 & 6,00 & 5,33 & 5,00 & 8,17 & 6,33 \\
\hline $\mathrm{C} 4$ & 5,33 & 7,33 & 5,17 & 6,67 & 5,33 & 4,00 & 7,33 & 5,00 \\
\hline $\mathrm{C} 5$ & 4,83 & 8,50 & 6,17 & 5,17 & 5,83 & 6,50 & 9,00 & 6,50 \\
\hline C6 & 6,17 & 6,17 & 5,33 & 5,50 & 6,50 & 9,00 & 6,50 & 6,00 \\
\hline $\mathrm{C} 7$ & 5,67 & 6,50 & 5,00 & 6,33 & 8,33 & 6,00 & 7,67 & 7,50 \\
\hline C8 & 6,33 & 3,50 & 5,17 & 5,50 & 5,83 & 4,50 & 5,00 & 6,17 \\
\hline C9 & 6,17 & 5,00 & 4,17 & 5,67 & 5,50 & 4,67 & 5,17 & 3,83 \\
\hline C10 & 7,17 & 6,67 & 6,17 & 6,83 & 7,67 & 6,17 & 7,00 & 5,83 \\
\hline C11 & 7,50 & 4,67 & 5,00 & 7,33 & 7,17 & 5,00 & 5,50 & 6,67 \\
\hline $\mathrm{C} 12$ & 5,00 & 7,50 & 5,67 & 5,00 & 4,67 & 5,50 & 8,17 & 6,00 \\
\hline C13 & 7,33 & 5,17 & 4,67 & 7,67 & 7,33 & 4,83 & 5,17 & 7,67 \\
\hline
\end{tabular}

Tabela 2. Relações entre habilitadores de customização e dimensões do serviço.

\begin{tabular}{|c|c|c|c|c|c|c|}
\hline & & & \multicolumn{4}{|c|}{ Dimensões do serviço } \\
\hline & & & Intangibilidade & Perecibilidade & Variabilidade & Inseparabilidade \\
\hline \multirow{8}{*}{$\begin{array}{l}\text { Habilitadores de } \\
\text { customizalção }\end{array}$} & \multicolumn{2}{|c|}{ Metodologia } & 3,40 & 4,60 & 4,60 & 3,80 \\
\hline & \multirow{5}{*}{ Processos } & Elicitação de ordem & 3,20 & 3,20 & 7,80 & 7,40 \\
\hline & & Postponement & 3,00 & 5,60 & 5,80 & 4,80 \\
\hline & & Plataformas de produtos & 3,00 & 3,60 & 4,80 & 4,40 \\
\hline & & Manufatura & 3,20 & 5,80 & 4,80 & 5,80 \\
\hline & & Cadeia de suprimentos & 3,20 & 5,60 & 3,80 & 4,80 \\
\hline & \multicolumn{2}{|c|}{ Sistemas e tecnologias de informação } & 4,40 & 5,00 & 8,00 & 7,20 \\
\hline & \multicolumn{2}{|c|}{ Tecnologias de manufatura } & 3,00 & 3,80 & 4,00 & 5,20 \\
\hline
\end{tabular}

Tabela 3. Características dos especialistas.

\begin{tabular}{cccc}
\hline Identificação & Formação & Área de atuação & Experiência na área (anos) \\
\hline Especialista 1 & Dr. Eng. Produção & Estratégia de produção & 12 \\
Especialista 2 & Dr. Eng. Produção & Desenvolvimento de produto & 7 \\
Especialista 3 & Dr. Eng. Produção & Gestão de serviços e manufatura & 18 \\
Especialista 4 & Dr. Administração & Marketing & 17 \\
Especialista 5 & Dr. Administração & Gestão de serviços & 8 \\
Especialista 6 & Dr. Administração & Marketing & 4 \\
Especialista 7 & Ms. Eng. Produção & Estratégia de produção & 3 \\
Especialista 8 & Ms. Administração & Gestão de serviços e marketing & 7 \\
\hline
\end{tabular}

O QFD é um método de desenvolvimento de produtos originado no Japão no início dos anos 1960. Duas abordagens para implementação do QFD encontram-se difundidas na literatura: o modelo de Akao (1996) e o modelo do ASI (American Supplier Institute), apresentado por Cohen (1995) e usado no presente trabalho.

0 modelo do ASI consiste de quatro matrizes interrelacionadas, todas seguindo uma mesma lógica de análise. Considere uma matriz apresentando $l$ itens nas linhas (por exemplo, características de customização do serviço), com pesos de importância organizados em um vetor $w$ de dimensão $(l \times 1)$, com elementos $w_{j}$ e $J$ itens nas colunas (por exemplo, habilitadores de customização). Na parte central da matriz, onde linhas e colunas se interseccionam, analistas são solicitados a avaliar o impacto dos itens nas colunas sobre os itens nas linhas, normalmente usando uma escala numérica de 0 a 9 ou de 0 a 5 . Seja $r_{i j}$ o impacto do item na $j$-ésima coluna sobre o item 
na $i$-ésima linha, correspondendo ao elemento $(i, j)$ de uma matriz de relacionamentos $\mathrm{R}$ de dimensão $(I \times J)$. Ao analisar-se uma matriz do QFD, deseja-se obter como resultado um vetor de prioridades, de dimensão $(J \times 1)$, para os itens nas colunas; esse vetor é designado por $\mathbf{p}$ e dado por:

$\mathrm{p}=\mathrm{R}^{t} \mathrm{w}$

onde $\mathbf{R}^{t}$ designa a transposta de $\mathbf{R}$.

As operações com as matrizes do QFD evoluem da primeira à última matriz. A informação é transferida ao longo dessas operações, de tal forma que a última matriz resume os resultados da análise feita em todas as matrizes anteriores.

$\mathrm{Na}$ análise reversa do QFD (FOGLIATTO; DA SILVEIRA, 2008), o objetivo é recuperar o vetor w a partir de $\mathrm{p}$ e $\mathrm{R}$ informados pelo analista. Tal operação reversa pode ser realizada aplicando-se a equação abaixo a uma dada matriz do QFD:

$\hat{\mathbf{w}}=\left(\mathbf{R R}^{t}\right)^{-1} \mathbf{R p}$

onde $\left(\mathbf{R R}^{t}\right)^{-1} \mathbf{R}$ é a pseudo inversa de R. A pseudo inversa na Equação 2 permite recuperar o vetor $\mathrm{w}$ em casos em que $R$ não é uma matriz quadrada (quando $R$ é uma matriz quadrada, $\left.\mathbf{R}^{-1}=\left(\mathbf{R R}^{t}\right)^{-1} \mathbf{R}\right)$. A Equação 2 restaura $\mathrm{w}$ sem nenhum erro sempre que $\mathbf{R}$ for uma matriz inversível. Caso contrário, a recuperação de $\mathrm{w}$ leva a um vetor com um erro residual acumulado em seu último elemento; quanto mais próximo $\mathrm{R}$ for de uma matriz quadrada, menor o erro residual.
No QFD reverso interessa a situação onde o vetor de pesos de prioridades obtido através de operações normais em uma dada matriz do QFD é modificado para refletir alguma situação de interesse. Seja $\tilde{\mathbf{p}}$ o vetor de prioridades modificadas. Substituindo $\mathrm{p}$ por $\tilde{\mathbf{p}}$ na Equação 2, a recuperação de $\mathrm{w}$ não ocorre, mas obtém-se um novo vetor $\hat{\mathbf{w}}$ de pesos que reflete as modificações representadas pelo vetor $\tilde{\mathbf{p}}$. Confrontando-se os vetores $\mathrm{w}$ e $\hat{\mathbf{w}}$ é possível avaliar os efeitos dos ajustes em $\mathbf{p}$ sobre os pesos originais. Uma situação similar é apresentada nos estudos de caso da seção 4 do presente artigo. A Figura 1 mostra a estrutura proposta.

\section{Resultados}

\subsection{Descrição dos casos}

Os casos usados para testar o modelo são de áreas de serviços distintas. 0 primeiro caso foi realizado no setor de obras de uma fornecedora de energia elétrica. Esse setor caracteriza-se pelo alto volume de obras executadas mensalmente e pela customização de cada uma delas. Por exemplo, em um mesmo mês existem obras de construção de uma subestação para um distrito industrial e, ao mesmo tempo, a instalação de um poste de luz em uma área residencial. Essas obras são customizadas a partir de um grupo de 2 mil componentes, previstos em legislação nacional e em normas específicas para o setor. Além do gerente de obras, responsável por aprovar e controlar todas as etapas do processo, dois coordenadores de obras participaram da realização desse estudo de caso.
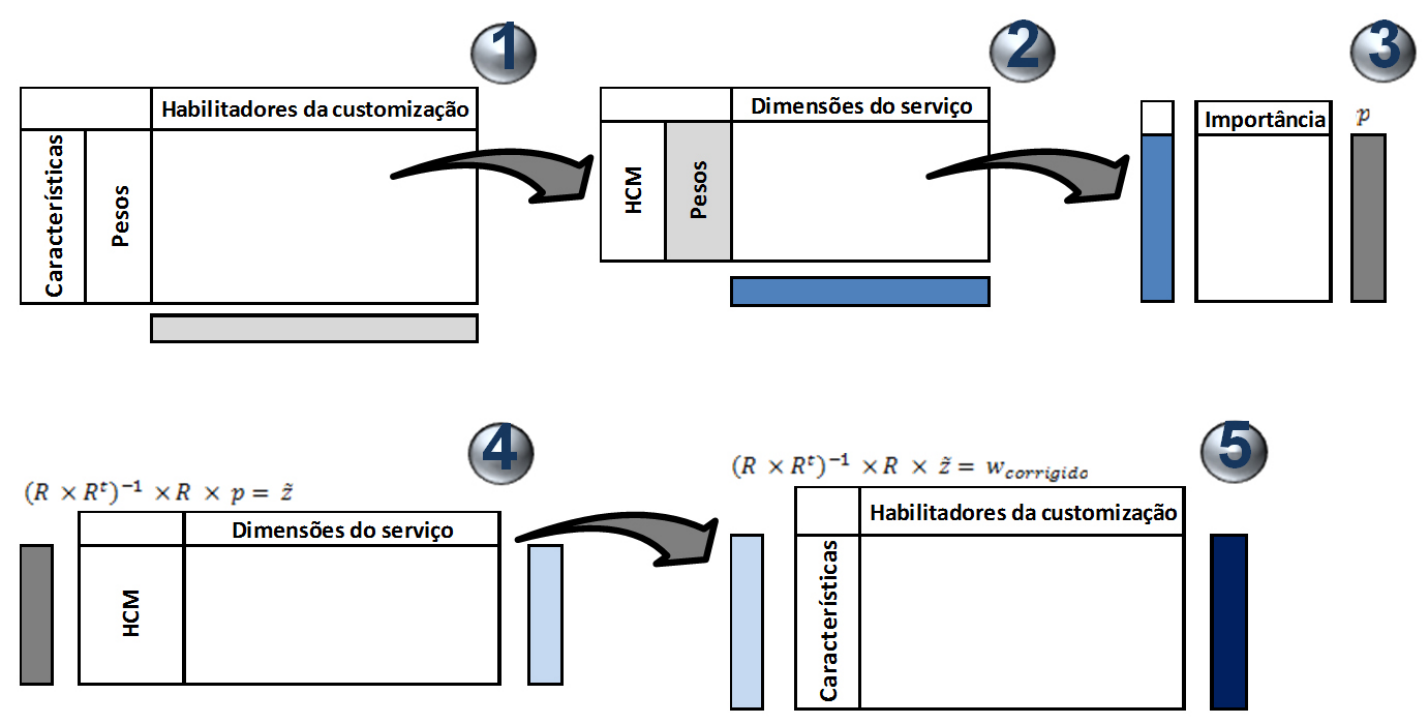

Figura 1. Diagrama de operações do QFD e do QFD reverso. 
0 segundo caso foi realizado em uma universidade, especificamente para entender a customização de uma área de ensino de cursos de curta duração e aperfeiçoamento. Esses cursos são normalmente indicados a profissionais que finalizaram o seu bacharelado ou licenciatura e necessitam de atualização de conhecimentos em curto espaço de tempo (menor que seis meses). Nessa universidade, esses cursos são modelados a partir de padrões de oferta, variando conforme a área de conhecimento, mas focados na manutenção de um volume semestral. Nesse caso, os participantes na pesquisa foram o responsável pelos cursos de curta duração da universidade e duas vendedoras dos cursos, que recebem e classificam as demandas dos clientes.

Em ambos os casos utilizaram-se as mesmas etapas. Primeiramente, a avaliação das CCMs foi coletada nas empresas através de entrevista e gravação dos dados com os participantes anteriormente mencionados. Nesse sentido, solicitou-se a avaliação do grau de importância de cada CCM e a importância de cada uma das dimensões de serviço. Na sequência, as informações foram repassadas para o modelo criado, possibilitando visualizar o papel de cada dimensão de serviço com relação às CCMs. Para tanto, foi realizado o procedimento do QFD reverso a partir de cada dimensão de serviço reponderada pela organização respondente.

Os dados coletados no primeiro e no segundo caso estão apresentados na Tabela 4. Os dados foram usados para iniciar a operacionalização do QFD e, posteriormente, levaram à identificação das características mais importantes de $\mathrm{CM}$ em serviços.

No primeiro caso, as características notadamente mais importantes são C2, C3, C5 e C10 e as menos importantes, C1, C4 e C6. Contudo, para o segundo caso, as características mais importantes são C3 e C4 e as menos importantes, C11 e C9. A opinião das empresas mostra que não é possível fazer uma previsão sobre a importância das características para customização de serviços, o que justifica a necessidade de uma sistemática para direcionar a customização do serviço.

\section{Discussão}

No primeiro caso, a empresa informou as dimensões de variabilidade e inseparabilidade como sendo as características de serviços mais importantes. Através dessa definição foi possivel ponderar os critérios para reversão, como mostra a Tabela 5 .

$\mathrm{Na}$ reversão foi possível verificar mudança de posição em boa parte das características; os resultados estão apresentados no Quadro 2. Ressalta-se que não é importante a ordem das características determinadas no QFD reverso, mas o quanto elas variaram em relação à posição inicial definida pela nota da empresa (vide Tabela 4). Dessa forma, o Quadro 2 mostra um comparativo da classificação atribuída inicialmente pela empresa e a posição final.

No caso, as características C2 e C5 já eram apontadas como as mais importantes pela empresa. Entretanto, as características C3 e C10, inicialmente ponderadas como importantes na classificação final, aparecem, respectivamente, na $10^{\mathrm{a}}$ e $7^{\mathrm{a}}$ posições no QFD reverso. Isso mostra que o julgamento inicial da organização não estava alinhado com as dimensões de serviços que a organização prioriza, o que nesse caso faz sentido: C3 refere-se ao total atendimento das necessidades do cliente, o que remeteria mais para uma

Tabela 4. Informações coletadas nas empresas usadas no passo 1.

\begin{tabular}{|c|c|c|c|}
\hline & \multirow{2}{*}{ Características de customização em massa } & \multicolumn{2}{|c|}{ Importância para a empresa respondente } \\
\hline & & Caso 1 & Caso 2 \\
\hline $\mathrm{C} 1$ & $\begin{array}{l}\text { Estrutura/arquitetura de serviços organizada em famílias, plataformas, módulos e } \\
\text { componentes. }\end{array}$ & 5,00 & 5,00 \\
\hline $\mathrm{C} 2$ & Projetos adaptáveis obtidos por processos customizados. & 10,00 & 7,00 \\
\hline C3 & $\begin{array}{l}\text { Integração do cliente no processo de serviços, permitindo total atendimento de suas } \\
\text { necessidades e expectativas. }\end{array}$ & 10,00 & 8,00 \\
\hline $\mathrm{C} 4$ & Planejamento de marketing e do marketing do serviço. & 3,00 & 8,00 \\
\hline C5 & Sistemas de informação que funcionem como canais de comunicação. & 10,00 & 7,00 \\
\hline C6 & Customização da cadeia de suprimentos. & 6,00 & 5,00 \\
\hline C7 & Gestão de dados, configurações, etapas da prestação de serviço. & 9,00 & 5,00 \\
\hline $\mathrm{C} 8$ & Determinação de custos por atividades. & 7,00 & 4,00 \\
\hline C9 & Integração entre planejamento dos serviços e as metas da organização. & 8,00 & 5,00 \\
\hline C10 & Agilidade e velocidade da prestação de serviços. & 10,00 & 5,00 \\
\hline C11 & $\begin{array}{l}\text { Ferramentas (engenharia concorrente, sistemas híbridos etc.) e estratégias de produção } \\
\text { (produção enxuta, produção em massa etc.). }\end{array}$ & 7,00 & 2,00 \\
\hline $\mathrm{C} 12$ & Criação de conhecimento através de retroalimentação de informações. & 8,00 & 5,00 \\
\hline C13 & Técnicas de repetição, padronização, prototipagem e personalização. & 9,00 & 7,00 \\
\hline
\end{tabular}


Tabela 5. Aplicação do método para o caso 1.

\begin{tabular}{|c|c|c|c|c|c|c|c|c|c|}
\hline \multirow{3}{*}{ Caracteristica } & \multirow{3}{*}{ Peso } & \multicolumn{8}{|c|}{ Habilitadores de customização } \\
\hline & & \multirow[b]{2}{*}{ Metodologias } & \multicolumn{5}{|c|}{ Processos } & \multirow[b]{2}{*}{ Sl e Tl } & \multirow{2}{*}{$\begin{array}{c}\text { Tecnologias } \\
\text { de } \\
\text { manufatura }\end{array}$} \\
\hline & & & $\begin{array}{l}\text { Elicitação de } \\
\text { ordem }\end{array}$ & Postponement & $\begin{array}{l}\text { Plataforma } \\
\text { de produtos }\end{array}$ & Manufatura & $\begin{array}{c}\text { Cadeia de } \\
\text { suprimentos }\end{array}$ & & \\
\hline $\mathrm{C} 1$ & 5 & 25,83 & 38,33 & 32,50 & 40,00 & 35,00 & 32,50 & 34,17 & 33,33 \\
\hline C2 & 10 & 70,00 & 66,67 & 70,00 & 78,33 & 71,67 & 73,33 & 68,33 & 68,33 \\
\hline C3 & 10 & 53,33 & 86,67 & 65,00 & 60,00 & 53,33 & 50,00 & 81,67 & 63,33 \\
\hline C4 & 3 & 16,00 & 22,00 & 15,50 & 20,00 & 16,00 & 12,00 & 22,00 & 15,00 \\
\hline C5 & 10 & 48,33 & 85,00 & 61,67 & 51,67 & 58,33 & 65,00 & 90,00 & 65,00 \\
\hline C6 & 6 & 37,00 & 37,00 & 32,00 & 33,00 & 39,00 & 54,00 & 39,00 & 36,00 \\
\hline C7 & 9 & 51,00 & 58,50 & 45,00 & 57,00 & 75,00 & 54,00 & 69,00 & 67,50 \\
\hline C8 & 7 & 44,33 & 24,50 & 36,17 & 38,50 & 40,83 & 31,50 & 35,00 & 43,17 \\
\hline C9 & 8 & 49,33 & 40,00 & 33,33 & 45,33 & 44,00 & 37,33 & 41,33 & 30,67 \\
\hline C10 & 10 & 71,67 & 66,67 & 61,67 & 68,33 & 76,67 & 61,67 & 70,00 & 58,33 \\
\hline C11 & 7 & 52,50 & 32,67 & 35,00 & 51,33 & 50,17 & 35,00 & 38,50 & 46,67 \\
\hline $\mathrm{C} 12$ & 8 & 40,00 & 60,00 & 45,33 & 40,00 & 37,33 & 44,00 & 65,33 & 48,00 \\
\hline \multirow[t]{2}{*}{$\mathrm{C} 13$} & 9 & 66,00 & 46,50 & 42,00 & 69,00 & 66,00 & 43,50 & 46,50 & 69,00 \\
\hline & & 625,33 & 664,50 & 575,17 & 652,50 & 663,33 & 593,83 & 700,83 & 644,33 \\
\hline
\end{tabular}

\begin{tabular}{cccccc}
\hline \multirow{2}{*}{ HCM } & \multirow{2}{*}{ Peso } & \multicolumn{4}{c}{ Dimensões do serviço } \\
\cline { 3 - 5 } & & Intangibilidade & Perecibilidade & Variabilidade & Inseparabilidade \\
\hline MET & 625,33 & $2.126,13$ & $2.876,53$ & $2.876,53$ & $2.376,27$ \\
ELO & 664,50 & $2.126,40$ & $2.126,40$ & $5.183,10$ & $4.917,30$ \\
POST & 575,17 & $1.725,50$ & $3.220,93$ & $3.335,97$ & $2.760,80$ \\
PP & 652,50 & $1.957,50$ & $2.349,00$ & $3.132,00$ & $2.871,00$ \\
MAN & 663,33 & $2.122,67$ & $3.847,33$ & $3.184,00$ & $3.847,33$ \\
CS & 593,83 & $1.900,27$ & $3.325,47$ & $2.256,57$ & $2.850,40$ \\
SI T1 & 700,83 & $3.083,67$ & $3.504,17$ & $5.606,67$ & $5.046,00$ \\
TM & 644,33 & $1.933,00$ & $2.448,47$ & $2.577,33$ & $3.350,53$ \\
& & $16.975,13$ & $23.698,30$ & $28.152,17$ & $28.019,63$ \\
\hline
\end{tabular}

\begin{tabular}{cc}
\hline$p$ & Importância \\
\hline $16.975,13$ & 0,50 \\
$23.698,30$ & 1,00 \\
$28.152,17$ & 2,00 \\
$28.019,63$ & 2,00 \\
\hline
\end{tabular}

\begin{tabular}{cc}
\hline & $\tilde{Z}$ \\
\hline MET & $-68,55$ \\
ELO & $-14,13$ \\
POST & $-32,39$ \\
PP & 119,25 \\
MAN & 14,60 \\
CS & $-2,48$ \\
SI / Tl & 1,63 \\
TM & $-15,59$ \\
\hline
\end{tabular}

\begin{tabular}{cc}
\hline & W corrigido \\
\hline C1 & 60,71 \\
C2 & 64,73 \\
C3 & $-73,18$ \\
C4 & $-634,55$ \\
C5 & 166,84 \\
C6 & $-225,31$ \\
C7 & $-61,03$ \\
C8 & $-262,02$ \\
C9 & 47,70 \\
C10 & 2,18 \\
C11 & 401,82 \\
C12 & 83,57 \\
C13 & $-49,34$ \\
\hline
\end{tabular}

estratégia de personalização do que propriamente de $\mathrm{CM}$; já $\mathrm{C} 10$ reflete a forma como a empresa gerencia a demanda, dependendo mais da capacidade de sequenciamento dos atendimentos do que das pessoas envolvidas na prestação do serviço. Por outro lado, as características C4 e C6, inicialmente apontadas pela empresa entre as menos importantes, confirmaram sua posição após a utilização do QFD reverso.
A característica $\mathrm{C}$, que não tinha importância na opinião inicial da empresa, cresceu para a $5^{a}$ posição na operacionalização do QFD reverso. Esse crescimento pode ser explicado pelo fato de a companhia tratar a inseparabilidade como um dos critérios mais importantes, o que necessariamente implica na organização da estrutura de produto para que essa dimensão do serviço seja contemplada. 
A característica $\mathrm{C} 12$, que subiu da $6^{\mathrm{a}}$ para a $3^{\mathrm{a}}$ posição, também merece destaque. A mudança de posição no ranking pode ser atribuída à nota dada pelos especialistas (vide Tabela 2), visto que as informações de customização podem ser usadas como elementos de aprendizagem quanto às demandas de clientes, algo que não é facilmente perceptível para uma organização.

No segundo caso, a dimensão do serviço definida pela universidade e abordada no caso como a mais importante foi a intangibilidade. A reversão vem mostrada na Tabela 6.

As mudanças de posição no ranking dada a realização do QFD reverso são perceptíveis, já que as quatro características definidas como mais importantes pela organização, após a utilização da metodologia, são outras, diferentes. No caso, as características C7, C9, C11 e C12 estão nas primeiras posições. A característica $\mathrm{C} 11$ é preponderante especialmente pela importância dada pelos especialistas, visto que no caso dessa universidade, utilizar ferramentas e estratégias de produção são aparentemente menos urgentes que outras mudanças. A característica C9 é muito importante para a organização, não tendo sido bem pontuada inicialmente. Porém fica claro que para a universidade planejar o serviço customizado uma das prioridades é alinhá-lo às metas organizacionais. 0 Quadro 3 traz o ranking das CCMs antes e depois da aplicação do QFD reverso no segundo estudo de caso.

Na sequência, a t3a posição é ocupada pela característica $\mathrm{C} 12$. Inicialmente não priorizada, a criação do conhecimento através de retroalimentação é importante para um ambiente de cursos de curta duração. A utilização das informações dos consumidores auxilia na definição de novas demandas, permitindo customização em maiores níveis e com maior abrangência. Outra característica que ascendeu posições foi a $\mathrm{C}$. Na verdade, era esperado que a

Quadro 2. Ranking para o caso 1.

\begin{tabular}{|c|c|c|c|l|}
\hline \multicolumn{2}{|c|}{ QFD reverso } & \multicolumn{2}{c|}{ NT empresa } & \multicolumn{1}{|c|}{ Caracteristicas } \\
\hline$-634,55$ & C4 & 3,00 & C4 & Planejamento de marketing e do marketing do serviço. \\
\hline$-262,02$ & C8 & 5,00 & C1 & Estrutura/arquitetura de serviços organizada em famílias, plataformas, módulos e componentes. \\
\hline$-225,30$ & C6 & 6,00 & C6 & Customização da cadeia de suprimentos. \\
\hline$-73,18$ & C3 & 7,00 & C8 & Determinação de custos por atividade. \\
\cline { 1 - 5 }$-61,02$ & C7 & 7,00 & C11 & $\begin{array}{l}\text { Ferramentas (engenharia concorrente, sistemas híbridos etc.) e estratégias de produção } \\
\text { (produção enxuta, produção em massa etc.). }\end{array}$ \\
\hline$-49,33$ & C13 & 8,00 & C9 & Integração entre planejamento dos serviços e as metas da organização. \\
\hline 2,18 & C10 & 8,00 & C12 & Criação de conhecimento através de retroalimentação de informações. \\
\hline 47,69 & C9 & 9,00 & C7 & Gestão de dados, configurações, etapas da prestação de serviços. \\
\hline 60,71 & C1 & 9,00 & C13 & Técnicas de repetição, padronização, prototipagem e personalização. \\
\hline 64,72 & C2 & 10,00 & C2 & Projetos adaptáveis obtidos por processos customizados. \\
\hline 83,56 & C12 & 10,00 & C3 & $\begin{array}{l}\text { Integração do cliente no processo de serviços, permitindo total atendimento de suas } \\
\text { necessidades e expectativas. }\end{array}$ \\
\hline 166,83 & C5 & 10,00 & C5 & Sistemas de informação que funcionem como canais de comunicação. \\
\hline 401,82 & C11 & 10,00 & C10 & Agilidade e velocidade da prestação de serviços. \\
\hline
\end{tabular}

Redução $\square$ Aumento $\square$ Mesma posição

Quadro 3. Ranking para o caso 2.

\begin{tabular}{|c|c|c|c|l|}
\hline \multicolumn{2}{|c|}{ QFD reverso } & \multicolumn{2}{|c|}{ NT empresa } & \multicolumn{1}{|c|}{ Características } \\
\hline$-15,88$ & C4 & 2,00 & C11 & $\begin{array}{l}\text { Ferramentas (engenharia concorrente, sistemas híbridos etc.) e estratégias de produção (produção } \\
\text { enxuta, produção em massa etc.). }\end{array}$ \\
\hline$-14,92$ & C8 & 4,00 & C8 & Determinação de custos por atividade. \\
\hline$-14,15$ & C10 & 5,00 & C1 & Estrutura/arquitetura de serviços organizada em famílias, plataformas, módulos e componentes. \\
\hline$-8,79$ & C13 & 5,00 & C6 & Customização da cadeia de suprimentos. \\
\hline$-7,35$ & C5 & 5,00 & C7 & Gestão de dados, configurações, etapas da prestação de serviços. \\
\hline$-6,82$ & C6 & 5,00 & C9 & lntegração entre planejamento dos serviços e as metas da organização. \\
\hline$-2,26$ & C2 & 5,00 & C10 & Agilidade e velocidade da prestação de serviços. \\
\hline 3,41 & C1 & 5,00 & C12 & Criação de conhecimento através de retroalimentação de informações. \\
\hline 7,32 & C3 & 7,00 & C2 & Projetos adaptáveis obtidos por processos customizados. \\
\hline 11,02 & C7 & 7,00 & C5 & Sistemas de informação que funcionem como canais de comunicação. \\
\hline 16,35 & C12 & 7,00 & C13 & Técnicas de repetição, padronização, prototipagem e personalização. \\
\hline 22,36 & C9 & 8,00 & C3 & $\begin{array}{l}\text { lntegração do cliente no processo de serviços, permitindo total atendimento de suas necessidades } \\
\text { e expectativas. }\end{array}$ \\
\hline 54,29 & C11 & 8,00 & C4 & Planejamento de marketing e do marketing do serviço. \\
\hline
\end{tabular}

Redução $\square$ Aumento $\square$ Mesma posição 
Tabela 6. Aplicação do método para o caso 2.

\begin{tabular}{|c|c|c|c|c|c|c|c|c|c|}
\hline \multirow{3}{*}{ Caracteristica } & \multirow{3}{*}{ Peso } & \multicolumn{8}{|c|}{ Habilitadores de customização } \\
\hline & & \multirow[b]{2}{*}{ Metodologias } & \multicolumn{5}{|c|}{ Processos } & \multirow[b]{2}{*}{ Sl e Tl } & \multirow{2}{*}{$\begin{array}{c}\text { Tecnologias } \\
\text { de } \\
\text { manufatura }\end{array}$} \\
\hline & & & $\begin{array}{c}\text { Elicitação de } \\
\text { ordem }\end{array}$ & Postponement & $\begin{array}{c}\text { Plataforma de } \\
\text { produtos }\end{array}$ & Manufatura & $\begin{array}{l}\text { Cadeia de } \\
\text { suprimentos }\end{array}$ & & \\
\hline $\mathrm{C} 1$ & 5 & 25,83 & 38,33 & 32,50 & 40,00 & 35,00 & 32,50 & 34,17 & 33,33 \\
\hline $\mathrm{C} 2$ & 7 & 49,00 & 46,67 & 49,00 & 54,83 & 50,17 & 51,33 & 47,83 & 47,83 \\
\hline C3 & 8 & 42,67 & 69,33 & 52,00 & 48,00 & 42,67 & 40,00 & 65,33 & 50,67 \\
\hline C4 & 8 & 42,67 & 58,67 & 41,33 & 53,33 & 42,67 & 32,00 & 58,67 & 40,00 \\
\hline C5 & 7 & 33,83 & 59,50 & 43,17 & 36,17 & 40,83 & 45,50 & 63,00 & 45,50 \\
\hline C6 & 5 & 30,83 & 30,83 & 26,67 & 27,50 & 32,50 & 45,00 & 32,50 & 30,00 \\
\hline C7 & 5 & 28,33 & 32,50 & 25,00 & 31,67 & 41,67 & 30,00 & 38,33 & 37,50 \\
\hline C8 & 4 & 25,33 & 14,00 & 20,67 & 22,00 & 23,33 & 18,00 & 20,00 & 24,67 \\
\hline C9 & 5 & 30,83 & 25,00 & 20,83 & 28,33 & 27,50 & 23,33 & 25,83 & 19,17 \\
\hline C10 & 5 & 35,83 & 33,33 & 30,83 & 34,17 & 38,33 & 30,83 & 35,00 & 29,17 \\
\hline C11 & 2 & 15,00 & 9,33 & 10,00 & 14,67 & 14,33 & 10,00 & 11,00 & 13,33 \\
\hline $\mathrm{C} 12$ & 5 & 25,00 & 37,50 & 28,33 & 25,00 & 23,33 & 27,50 & 40,83 & 30,00 \\
\hline \multirow[t]{2}{*}{$\mathrm{C} 13$} & 7 & 51,33 & 36,17 & 32,67 & 53,67 & 51,33 & 33,83 & 36,17 & 53,67 \\
\hline & & 436,50 & 491,17 & 413,00 & 469,33 & 463,67 & 419,83 & 508,67 & 454,83 \\
\hline
\end{tabular}

\begin{tabular}{cccccc}
\hline \multirow{2}{*}{ HCM } & \multirow{2}{*}{ Peso } & \multicolumn{3}{c}{ Dimensões do serviço } \\
\cline { 3 - 5 } & & Intangibilidade & Perecibilidade & Variabilidade & Inseparabilidade \\
\hline MET & 436,50 & $1.484,10$ & $2.007,90$ & $2.007,90$ & $1.658,70$ \\
ELO & 491,17 & $1.571,73$ & $1.571,73$ & $3.831,10$ & $3.634,63$ \\
POST & 413,00 & $1.239,00$ & $2.312,80$ & $2.395,40$ & $1.982,40$ \\
PP & 469,33 & $1.408,00$ & $1.689,60$ & $2.252,80$ & $2.065,07$ \\
MAN & 463,67 & $1.483,73$ & $2.689,27$ & $2.225,60$ & $2.689,27$ \\
CS & 419,83 & $1.343,47$ & $2.351,07$ & $1.595,37$ & $2.015,20$ \\
SI / Tl & 508,67 & $2.238,13$ & $2.543,33$ & $4.069,33$ & $3.662,40$ \\
TM & 454,83 & $1.364,50$ & $1.728,37$ & $1.819,33$ & $2.365,13$ \\
& & $12.132,67$ & $16.894,07$ & $20.196,83$ & $20.072,80$ \\
\hline
\end{tabular}

\begin{tabular}{cc}
\hline$p$ & Importância \\
\hline $24.265,33$ & 0,50 \\
$8.447,03$ & 0,50 \\
$10.098,42$ & 0,50 \\
$10.036,40$ & 2,00 \\
\hline
\end{tabular}

universidade priorizasse a gestão de suas opções e etapas na elaboração anteriormente à oferta de cursos.

Por outro lado, é interessante ver como as características C3, C4, C5 e C13 apresentaram decréscimo em sua posição. No caso de $\mathrm{C} 3$ pode-se explicar a queda em decorrência da classificação dos especialistas. Essa característica reflete um nível alto

\begin{tabular}{cc}
\hline & $\tilde{Z}$ \\
\hline MET & 42,76 \\
ELO & $-4,22$ \\
POST & $-53,28$ \\
PP & $-36,09$ \\
MAN & 16,24 \\
CS & $-4,79$ \\
SI / T1 & 25,68 \\
TM & 12,57 \\
\hline
\end{tabular}

\begin{tabular}{cc}
\hline & W corrigido \\
\hline C1 & 3,41 \\
C2 & $-2,26$ \\
C3 & 7,32 \\
C4 & $-15,88$ \\
C5 & $-7,35$ \\
C6 & $-6,82$ \\
C7 & 11,02 \\
C8 & $-14,92$ \\
C9 & 22,36 \\
C10 & $-14,15$ \\
C11 & 54,29 \\
C12 & 16,35 \\
C13 & $-8,79$ \\
\hline
\end{tabular}

de customização a ser ofertado pela empresa; assim, mesmo que a organização a considere importante, não há como priorizar essa característica em detrimento de outras. A característica C4 teve uma inversão completa em sua posição. lsso pode ser explicado, uma vez que essa característica é dependente da estratégia da organização. 
Tabela 7. Comparação de rankings.

\begin{tabular}{|c|c|c|c|}
\hline & \multirow[t]{2}{*}{ Caracteristicas de customização em massa } & \multicolumn{2}{|c|}{$\begin{array}{l}\text { Importância para implementar } \\
\text { serviços customizados }\end{array}$} \\
\hline & & Caso 1 & Caso 2 \\
\hline $\mathrm{C} 1$ & $\begin{array}{l}\text { Estrutura/arquitetura de serviços organizada em famílias, plataformas, módulos e } \\
\text { componentes. }\end{array}$ & $5^{\mathrm{a}}$ & $6^{\mathrm{a}}$ \\
\hline C2 & Projetos adaptáveis obtidos por processos customizados. & $4^{\mathrm{a}}$ & $7^{\mathrm{a}}$ \\
\hline C3 & $\begin{array}{l}\text { Integração do cliente no processo de serviços, permitindo total atendimento de suas } \\
\text { necessidades e expectativas. }\end{array}$ & $10^{\mathrm{a}}$ & $5^{\mathrm{a}}$ \\
\hline $\mathrm{C} 4$ & Planejamento de marketing e do marketing do serviço. & $13^{\mathrm{a}}$ & $13^{\mathrm{a}}$ \\
\hline C5 & Sistemas de informação que funcionem como canais de comunicação. & $2^{\mathrm{a}}$ & $9^{\mathrm{a}}$ \\
\hline C6 & Customização da cadeia de suprimentos. & $11^{\mathrm{a}}$ & $8^{\mathrm{a}}$ \\
\hline C7 & Gestão de dados, configurações, etapas da prestação de serviço. & $9^{a}$ & $4^{\mathrm{a}}$ \\
\hline C8 & Determinação de custos por atividade. & $12^{\mathrm{a}}$ & $12^{\mathrm{a}}$ \\
\hline C9 & Integração entre o planejamento dos serviços e as metas da organização. & $6^{a}$ & $2^{\mathrm{a}}$ \\
\hline C10 & Agilidade e velocidade da prestação de serviços. & $7^{\text {a }}$ & $11^{\mathrm{a}}$ \\
\hline C11 & $\begin{array}{l}\text { Ferramentas (engenharia concorrente, sistemas híbridos etc.) e estratégias de produção } \\
\text { (produção enxuta, produção em massa etc.). }\end{array}$ & $1^{\mathrm{a}}$ & $1^{\circ}$ \\
\hline C12 & Criação de conhecimento através de retroalimentação de informações. & $3^{\mathrm{a}}$ & $3^{\mathrm{a}}$ \\
\hline $\mathrm{C} 13$ & Técnicas de repetição, padronização, prototipagem e personalização. & $8^{\mathrm{a}}$ & $10^{\mathrm{a}}$ \\
\hline
\end{tabular}

A característica C5 apresentou um decréscimo porque, na opinião dos especialistas (ver Tabela 2), é tratada como interface entre o cliente e a organização. A classificação da empresa é consequência de uma interpretação na qual essa característica é entendida como porta de venda do serviço. Assim, entende-se por que a característica decresce de sua classificação inicial para uma posição inferior. Por fim, pode-se explicar a queda da característica $\mathrm{C} 13$ em função da importância dada pelos especialistas às questões de padronização e personalização. Na realidade, a característica padronização e personalização depende de uma estrutura de customização; essa característica é importante para tornar a customização em massa do serviço uma estratégia madura.

A análise dos casos revela que há características de customização em massa mais próximas da área de serviços, enquanto outras não são compatíveis, como apresentado na Tabela 7. Por exemplo, C11 e C12 são as mais próximas da área de serviços, enquanto C4 e C8 são mais distantes.

\section{Conclusões}

O objetivo do trabalho foi criar um método para projetar o serviço customizado em massa. Dessa forma, as variáveis $(l)$ características de customização em massa, (ii) habilitadores de customização em massa e (iii) habilitadores de serviços foram relacionadas através de um procedimento que utiliza o QFD e o QFD reverso.

Os resultados mostram que é possível aplicar o método proposto para duas situações. Primeiro, para avaliar quais características de CM são mais importantes para um serviço customizado já existente. Segundo, para projetar um serviço customizado, pressupondo que esse serviço não exista. Os casos utilizados refletem essas duas situações. No primeiro caso, o serviço de obras já é customizado e procedeu-se à sua avaliação. No segundo caso, há uma proposta de customização de cursos de curta duração.

A contribuição, no primeiro caso, está no fato de permitir que as melhorias sejam focadas naquilo que a empresa realmente considera importante. A utilização da metodologia estratifica as características mais importantes na visão da empresa e corrige essa análise a partir do habilitador de serviço entendido como o mais importante para um tipo de negócio.

A contribuição, no segundo caso, reside em sua originalidade - trata-se de uma proposição não encontrada na literatura. Através dela é possível inserir uma fase preliminar na customização do serviço, em que deficiências e prioridades podem ser identificadas, facilitando-se o entendimento das necessidades do cliente. Por exemplo, a estruturação de qualquer serviço customizado de manutenção poderá ser realizada pelo grupo de necessidades e especificações do cliente para um dado grupo de máquinas.

Por fim, pode-se dizer que a metodologia pode ser aplicada para projetar serviços customizados. Há uma base teórica que respalda as características, os habilitadores de customização em massa e os habilitadores de serviços. Além disso, não se identificou na literatura uma sistemática similar para projetar o serviço customizado. Alternativamente, poderia ser concebida uma sistemática para avaliar as fases de projeto e prestação do serviço customizado. 


\section{Referências}

ABDELKAFl, N. et al. NPD-SCM Alignment in Mass Customization. In: FOGLIATTO, F. S.; DA SILVEIRA, G. Mass Customization: Engineering and Managing Global Operations. Springer Velag, 2010.

AKAO, Y. Manual de aplicação do desdobramento da função qualidade (volume 1) - Introdução ao desdobramento da qualidade. Tradução de Zelinda Tomie Fujikawa, Seiichiro Takahashi. Belo Horizonte: UFMG, Escola de Engenharia, Fundação Cristiano Ottoni, 1996.

ALIZON, F. et al. Framework for product family design and development. Concurrent Engineering: Research and Applications, v. 15, n. 2, p. 187-199, 2007.

ANDERSON-CONNELL, J. J.; ULRICH, P. V.; BRANNON, E. L. A consumer-driven model for mass customization in the apparel market. Journal of Fashion Marketing Management, v. 6, n. 3, p. 240-258, 2002. http://dx.doi. org/10.1108/13612020210441346

APEAGYEI, P. R.; OTIENO, R. Usability of pattern customizing technology in the achievement and testing of fit for mass customisation. Journal of Fashion Marketing and Management, v. 11, n. 3, p. 349-365, 2007. http:// dx.doi.org/10.1108/13612020710763100

BARDAKCl, A.; WHITELOCK, J. Mass-customisation in marketing: the consumer perspective. Journal of Consumer Marketing, v. 20, n. 5, p. 463-479, 2003. http://dx.doi.org/10.1108/07363760310489689

BARDAKCl, A.; WHITELOCK, J. How "ready" are customers for mass customization? An exploratory investigation. European Journal of Marketing, v. 38, n. 11-12, p. 1396-1416, 2004. http://dx.doi. org/10.1108/03090560410560164

BARDAKCl, A.; WHITELOCK, J. A comparison of customers' readiness for mass-customisation: Turkish vs British customers. European Business Review, v. 17, n. 5, p. 397-410, 2005. http://dx.doi. org/10.1108/09555340510620311

BARE, M.; COX, J. J. Applying principles of mass customization to improve the empirical product development process. Journal of Intelligent Manufacturing, v. 19, p. 565576, 2008. http://dx.doi.org/10.1007/s10845-0080134-0

BASK, A. H.; TINNILÄ, M.; RAJAHONKA, M. Matching service strategies, business models and modular business processes. Business Process Management Journal, v. 16, n. 1, p. 153-180, 2010. http://dx.doi. org/10.1108/14637151011017994

BOOMS, B. H.; BITNER, M. J. Marketing Strategies and Organizational Structures for Service Firms. In: DONNELLY, J.; GEORGE, W. R. Marketing of Services. Chicago: American Marketing Association, 1981. p. 47-51.

BROWN, S.; BESSANT, J. The manufacturing strategycapabilities links in mass customisation and agile manufacturing - an exploratory study. International Journal of Operations \& Production Management, v. 23, n. 7, p. 707-730, 2003. http://dx.doi. org/10.1108/01443570310481522

BUFFINGTON, J. Comparison of mass customization and generative customization in mass markets. Industrial Management \& Data Systems, v. 111, n. 1, p. 41-62, 2011. http://dx.doi.org/10.1108/02635571111099721
CAO, J. et al. An interactive service customization model. Information and Software Technology, v. 48, p. 280-296, 2006. http://dx.doi.org/10.1016/j. infsof.2005.04.007

CHEN, Z.; WANG, L. A generic activity-dictionarybased method for product costing in mass customization. Journal of Manufacturing Technology Management, v. 18, n. 6, p. $678-700,2007$. http://dx.doi. org/10.1108/17410380710763859

CHO, H.; FlORITO, S. S. Acceptance of online customization for apparel shopping. International Journal of Retail \& Distribution Management, v. 37, n. 5, p. 389-407, 2009. http://dx.doi.org/10.1108/09590550910954892

COHEN, W. W. Pac-learning non-recursive prolog clauses. Artificial Intelligence, v. 79, n. 1, p. 1-38, 1995. http:// dx.doi.org/10.1016/0004-3702(94)00034-4

CORBETT, B.; ROSEN, D. W. A configuration design based method for platform commonization for product families. Artificial Intelligence for Engineering Design, Analysis and Manufacturing, v. 18, p. 21-39, 2004. http://dx.doi.org/10.1017/S089006040404003X

DA SILVEIRA, G.; BORENSTEIN, D.; FOGLIATTO, F. S. MasS customization: Literature review and research directions. International Journal of Production Economics, v. 72 , p. 1-13, 2001. http://dx.doi.org/10.1016/S09255273(00)00079-7

DAHMUS, J. B.; ZUGASTl, J. G. P.; OTTO, K. N. Modular product architecture. Designs Studies, v. 22, n. 5, p. 409-424, 2001. http://dx.doi.org/10.1016/S0142694X(01)00004-7

DAl, Z.; SCOTT, M. J. Product platform design through sensitivity analysis and cluster analysis. International Journal of Intelligent Manufacturing, v. 18, p. 97113, 2007. http://dx.doi.org/10.1007/s10845-007$0011-2$

DAVIS, S. M. From future perfect: Mass customizing. Planning Review, v. 17, p. 16-21, 1987. http://dx.doi. org/10.1108/eb054249

DEAN, P. R.; TU, Y. L.; XUE, D. An information system for one-of-a-kind producion. International Journal of Production Research, v. 47, n. 4, p. 1071-1087, 2009. http://dx.doi.org/10.1080/00207540701543593

DIETRICH, A. J.; KIRN, S.; SUGUMARAM, V. A service-oriented architecture for mass customization: a shoe industry case study. IEEE Transactions on Engineering Management, v. 54, n. 1, p. 190-204, 2007. http://dx.doi.org/10.1109/ TEM.2006.889076

DU, J.; JIAO, Y.-Y.; JIAO, J. Integrated BOM and routing generator for variety synchronization in assembly-toorder production. Journal of Manufacturing Technology Management, v. 16, n. 2, p. 233-243, 2005. http:// dx.doi.org/10.1108/17410380510576859

DURAY, R. Mass customization origins: mass or custom manufacturing? International Journal of Operations \& Production Management, v. 22, n. 3, p. 314-328, 2002. http://dx.doi.org/10.1108/01443570210417614

DURAY, R. et al. Approaches to mass customization: configurations and empirical validation. Journal of Operations Management, v. 18, p. 605-625, 2000. http:// dx.doi.org/10.1016/S0272-6963(00)00043-7

ENDO, S.; KINCADE, D. H. Mass customization for long-term relationship development: Why consumers purchase mass 
customized products again. Qualitative Market Research: An International Journal, v. 11, n. 3, p. 275-294, 2008.

FENG, Y. X. et al. An exploratory study of the general requirement representation model for product configuration in mass customization mode. International Journal of Advanced Manufacturing Technology, v. 40, p. 785-796, 2009. http://dx.doi.org/10.1007/s00170008-1381-0

FITZSIMMONS, J. A.; FITZSIMMONS, M. J. Administração de Serviços. Porto Alegre: Bookman, 2010.

FIXSON, S. K. Modularity and commonality research: past developments and future opportunities. Concurrent Engineering: Research and Applications, v. 15, n. 2, p. 85-110, 2007.

FOGLIATTO, F. S.; DA SILVEIRA, G. J. C.; BORENSTEIN, D. The mass customization decade: An updated review of the literature. International Journal of Production Economics, v. 138, p. 14-25, 2012. http://dx.doi. org/10.1016/j.jppe.2012.03.002

FOGLIATTO, F. S.; DA SILVEIRA, G. J. C. Mass customization: A method for market segmentation and choice menu design. International Journal of Productions Economics, v. 111, p. 606-622, 2008. http://dx.doi.org/10.1016/j. ijpe.2007.02.034

GERSHENSON, J. K.; PRASAD, G. J.; ZHANG, Y. Product modularity: definitions and benefits. Journal of Engineering Design, v. 14, n. 3, p. 295-313, 2003. http:// dx.doi.org/10.1080/0954482031000091068

GERSHENSON, J. K. Product modularity: measures and design methods. Journal of Engineering Design, v. 15, n. 1, p. 33-51, 2004. http://dx.doi. org/10.1080/0954482032000101731

GOTTFRIDSSON, P. Development of personalized services in small business: an iterative learning process. Managing Service Quality, v. 20, n. 4, p. 388-400, 2010. http:// dx.doi.org/10.1108/09604521011057504

GRENCl, R. T.; WATTS, C. A. Maximizing customer value via mass customized e-consumer services. Business Horizons, v. 50, p. 123-132, 2007. http://dx.doi. org/10.1108/09604521011057504

HAUG, A.; LADEBY, K.; EDWARDS, K. From engineer-toorder to mass customization. Management Research News, v. 32, n. 7, p. 633-644, 2009. http://dx.doi. org/10.1108/01409170910965233

HELO, P. T. et al. Integrated Vehicle Configuration System. - Connecting the domains of mass customization. Computer in Industry, v. 61, p. 44-52, 2010. http:// dx.doi.org/10.1016/j.compind.2009.07.006

HELMS, M. M. et al. Technologies in support of mass customization strategy: Exploring the linkages between e-commerce and knowledge management. Computers in Industry, v. 59, p. 351-363, 2008. http://dx.doi. org/10.1016/j.compind.2007.09.003

HOEK, R. l. V. The rediscovery of postponement a literature review and directions for research. Journal of Operations Management, v. 19, p. 161-184, 2001. http://dx.doi. org/10.1016/S0272-6963(00)00057-7

HUANG, G. Q.; ZHANG, X. Y.; LO, V. H. Y. Integrated configuration of platform products and supply chains for mass customization: a game-theoretic approach. IEEE Transactions on engineering management, v. 54, n. 1, p. 156-171, 2007. http://dx.doi.org/10.1109/ TEM.2006.889074
HUANG, G. Q.; ZHANG, X. Y.; LIANG, L. Towards integrated optimal configuration of platform products, manufacturing processes, and supply chains. Journal of Operations Management, v. 23, p. 267-290, 2005. http:// dx.doi.org/10.1016/j.jom.2004.10.014

ISMAlL, $H$. et al. How Small and Medium Enterprises Effectively Participate in the Mass Customization Game. IEEE Transactions on engineering management, v. 54, n. 1, p. 88-97, 2007. http://dx.doi.org/10.1109/ TEM.2006.889069

JIAO, J.; MA, Q.; TSENG, M. M. Towards high value-added products and services: mass customization and beyond. Technovation, v. 23, p. 809-821, 2003. http://dx.doi. org/10.1016/S0166-4972(02)00023-8

JIAO, J.; SIMPSON, T. W.; SIDDIQUE, Z. Product family design and platform-based product development: a state-ofthe-art review. Journal of Intelligent Manufacturing, v. 18, p. 5-29, 2007. http://dx.doi.org/10.1007/s10845007-0003-2

JIAO, J.; TSENG, M. M. A methodology of developing product family architecture for mass customization. Journal of Intelligent Manufacturing, v. 10, p. 3-20, 1999. http:// dx.doi.org/10.1023/A:1008926428533

JIAO, J.; TSENG, M. M. Customizability analysis in design for mass customization. Computer Aided Design, v. 36, p. 745-757, 2004. http://dx.doi.org/10.1016/j. cad.2003.09.012

JIN, L.; HE, Y.; SONG, H. Service customization: To upgrade or to downgrade? An investigation of how option framing affects tourists' choice of package-tour services. Tourism Management, p. 1-10, 2011.

JITPAIBOON, T.; DANGOL, R.; WALTERS, J. The study of cooperative relationships and mass customization. Management Research News, v. 32, n. 9, p. 804-815, 2009. http://dx.doi.org/10.1108/01409170910980326

JOSE, A.; TOLLENAERE, M. Modular and platform methods for product family design: literature analysis. Journal of Intelligent Manufacturing, v. 16, p. 371-390, 2005. http://dx.doi.org/10.1007/s10845-005-7030-7

KARLSSON, A. Assembly-initiated production - a strategy for mass-customization utilizing modular, hybrid automatic production systems. Assembly Automation, v. 22, n. 3, p. 239-247, 2002. http://dx.doi. org/10.1108/01445150210436455

KINCADE, D. H.; REGAN, C.; GIBSON, F. Y. Concurrent engineering for product development in mass customization for the apparel industry. International Journal of Operations \& Production Management, v. 27, n. 6, p. 627-649, 2007. http://dx.doi. org/10.1108/01443570710750295

KOTHA, S. Mass Customization: Implementing the Emerging Paradigm for Competitive Advantage. Strategic Management Journal, v. 16, p. 21-42, 1995. http:// dx.doi.org/10.1002/smj.4250160916

KOTLER, P.; KELLER, K. L. Administração de Marketing. São Paulo: Pearson, 2006.

KUMAR, A. From mass customization to mass personalization: a strategic transformation. International Journal of Flexibility and Manufacturing System, v. 19, p. 533547, 2008. http://dx.doi.org/10.1007/s10696-0089048-6

KUMAR, A.; GATOUFl, S.; REISMAN, A. Mass customization research: trends, directions, diffusion intensity, and 
taxonomic frameworks. International Journal of Flexibility and Manufacturing System, v. 19, p. 637665 , 2008. http://dx.doi.org/10.1007/s10696-0089051-y

LEE, N. K. S.; DAl, J. B. Designing and Planning of Material Handling Systems for Mass Customization. In: FOGLIATTO, F. S.; DA SILVEIRA, G. Mass Customization: Engineering and Managing Global Operations. Springer Velag, 2010.

LEVITT, T. A imaginação de Marketing. São Paulo: Atlas, 1991.

LI, Y.; XUE, D.; GU, P. Design for Product Adaptability. Concurrent Engineering,v. 16, n. 3, p. 221-232, Sept. 2008. http://dx.doi.org/10.1177/1063293X08096178

LINDQUIST, A.; BERGLUND, F.; JOHANNESSON, $H$. Supplier Integration and Communication Strategies in Collaborative Platform Development. Concurrent Engineering, v. 16, n. 1, p. 23-35, 2008. http://dx.doi. org/10.1177/1063293X07084639

LIOU, J. J. H.; YEN, L.; TZENG, G. H. Using decision rules to achieve mass customization of airline services. European Journal of Operations Research, v. 205, p. 680686, 2010. http://dx.doi.org/10.1016/j.ejor.2009.11.019

MA, Y.; JIAO, J.; DENG, Y. Web Service-oriented Electronic Catalogs for Product Customization. Concurrent Engineering, v. 16, n. 4, p. 263-270, 2008. http://dx.doi. org/10.1177/1063293X08100026

MARION, T. J. et al. Design for mass customization in the early stages of product development. International Design Engineering Technical Conferences \& Computers and Information in Engineering Conference, p. 1013, 2006.

McCARTHY, 1. P.; PITT, L.; BERTHON, P. Service Customization Through Dramaturgy. In: FOGLIATTO, F. S.; DA SILVEIRA, G. Mass Customization: Engineering and Managing Global Operations. Springer Velag, 2010.

MUFFATTO, M. Introducing a platform strategy in product development. International Journal of Production Economics, v. 60-61, p. 145-153, 1999. http://dx.doi. org/10.1016/S0925-5273(98)00173-X

MUN, D. et al. Engineered-to-order Approach for Providing Flexibility in e-Commerce of Mold Parts. Concurrent Engineering, v. 15, n. 4, p. 345-355, 2007. http://dx.doi. org/10.1177/1063293X07084634

NI, Q. F.; LU, W. F.; YARLAGADDA, P. K. D. V. An Extensible Product Structure Model for Product Lifecycle Management in the Make-to-Order Environment. Concurrent Engineering, v. 16, n. 4, p. 243-251, 2008. http://dx.doi.org/10.1177/1063293X08100023

NIELSEN, K. J.; COX, J. J. Implementation of Biomechanical Mating Conditions in CAD. Computer Aided Design and Applications, v. 5, n. 1-4, p. 338-353, 2008.

ORDANINI, A. et al. Crowd-funding: transforming customers into investors through innovative service platforms. Journal of service management, v. 22, n. 4, p. 443470, 2011.

O'SHAUGHNESSY, J.; O'SHAUGHNESSY, N. J. The servicedominant perspective: a backward step?. European Journal of Marketing, v. 43, n. 5-6, p. 784-793, 2009. http://dx.doi.org/10.1108/03090560910947043

PAN, B.; HOLLAND, R. A mass customised supply chain for the fashion system at the design-production interface. Journal of Fashion Marketing and Management, v. 10, n. 3, p. 345-359, 2006. http://dx.doi. org/10.1108/13612020610679312

PETERS, L.; SAIDIN, H. IT and the mass customization of services: the challenge of implementation. International Journal of Information Management, v. 20, p. 103119, 2000. http://dx.doi.org/10.1016/S02684012(99)00059-6

PILLER, F. T.; MOESLEIN, K.; STOTKO, C. M. Does mass customization pay? An economic approach to evaluate customer integration. Production Planning and Control, v. 15, n. 4, p. 435-444, 2004. http://dx.doi. org/10.1080/0953728042000238773

PILlER, F. T. Mass Customization: Reflections on the State of the Concept. International Journal of Flexible Manufacturing Systems, v. 16, p. 313-334, 2004. http:// dx.doi.org/10.1007/s10696-005-5170-x

PILLER, F. T. Observations on the present and future of mass customization. International Journal of Flexibility and Manufacturing System, v. 19, p. 630-636, 2008. http:// dx.doi.org/10.1007/s10696-008-9042-Z

SALVADOR, F.; MARTIN, P.; PILLER, F. Decodificando a customização em massa. HSM Management, v. 6, n. 77, p. 140-148, 2009.

SALVADOR, F.; FORZA, C.; RUNGTUSANATHAM, M. Modularity, product variety, production volume, and component sourcing: theorizing beyond generic prescriptions. Journal of Operations Management, v. 20, p. 549-575, 2002. http://dx.doi.org/10.1016/S02726963(02)00027-X

SHAMSUZZOHA, A.; KYLLÖNEN, S.; HELO, P. Collaborative customized product development framework. Industrial Management and Data System, v. 109, n. 5, p. 718-735, 2009. http://dx.doi. org/10.1108/02635570910957678

SIDDIQUE, Z.; BODDU, K. R. A mass customization information framework for integration of customer in the configuration/design of a customized product. Artificial Intelligence for Engineering Design, Analysis and Manufacturing, v. 18, p. 71-85, 2004. http://dx.doi. org/10.1017/S0890060404040065

SIGALA, M. Mass customization implementation models and customer value in mobile phones services: Preliminary findings from Greece. Managing Service Quality, v. 16, n. 4, p. 395-420, 2006. http://dx.doi. org/10.1108/09604520610675720

SIMPSON, T. W. et al. Platform-Based Design And Development: Current Trends And Needs In Industry. International Design Engineering Technical Conferences \& Computers and Information in Engineering Conference, 2006.

SIMPSON, T. W.; MAIER, J. R. A.; MISTREE, F. Product Platform design: method and application. Research Engineering Design, v. 13, p. 2-22, 2001. http://dx.doi. org/10.1007/s001630100002

SIMPSON, T. W. Product platform design and customization: Status and promise. Artificial Intelligence for Engineering Design, Analysis and Manufacturing, v. 18, p. 3-20, 2004. http://dx.doi.org/10.1017/S0890060404040028

SPRING, M.; ARAUJO, L. Service, services and products: rethinking operations strategy. International Journal of Operations \& Production Management, v. 29, n. 5, p. 444-467, 2009. http://dx.doi. org/10.1108/01443570910953586 
STARR, M. K. Modular production - a 45-year-old concept. International Journal of Operations \& Production Management, v. 30, n. 1, p. 7-19, 2010. http://dx.doi. org/10.1108/01443571011012352

TANG, A. et al. A comparative study of architecture knowledge management tools. The Journal of Systems and Software, v. 83, p. 352-370, 2010. http://dx.doi. $\operatorname{org} / 10.1016 /$ j.jss.2009.08.032

THEVENOT, H. J.; SIMPSON, T. W. Commonality indices for product family design: a detailed comparison. Journal of Engineering Design, v. 17, n. 2, p. 99-119, 2006. http:// dx.doi.org/10.1080/09544820500275693

TSENG, M. M.; JIA0, J. Design for Mass Customization. Annals of the CIRP, v. 45, n. 1, p. 153-156, 1996. http:// dx.doi.org/10.1016/S0007-8506(07)63036-4

TSENG, M. M.; RADKE, A. M. Production Planning and Control for Mass Customization. In: FOGLIATTO, F. S.; DA SILVEIRA, G. Mass Customization: Engineering and Managing Global Operations. Springer Velag, 2010.

WANG, H.; LIN, Z. Defects tracking matrix for mass customization production based on house of quality. International Journal of Flexibility and Manufacturing System, v. 19, p. 666-684, 2008. http://dx.doi. org/10.1007/s10696-007-9025-5

WILlIAMS, C. B. et al. Designing Platforms for Customizable Products and Processes in Markets of Non-Uniform Demand. Concurrent Engineering, v. 15, n. 2, p. 201-216, 2007. http://dx.doi. org/10.1177/1063293X07079328

WITELL, L. et al. Idea generation: customer co-creation versus traditional market research techniques. Journal of Service Management, v. 22, n. 2, p. 140-159, 2011. http://dx.doi.org/10.1108/09564231111124190

YANG, B.; BURNS, N. D.; BACKHOUSE, C. J. Postponement: a review and an integrated framework. International Journal of Operations \& Production Management, v. 24, n. 5, p. $468-487,2004$. http://dx.doi. org/10.1108/01443570410532542

ZAHAJ, D.; GRIFFIN, A. Are customer information systems worth it? Results from B2B services. Marketing Science Institute, 2002. Working Paper.

ZEITHAML, V. A. How consumer evaluation process differ betwenn goods and services. In: DONNELLY, J.; GEORGE, W. R. Marketing of Services. Chicago: American Marketing Association, 1981. p. 186-190.

ZHA, X. F.; SRIRAM, R. D.; LU, W. F. Evaluation and selection in product design for mass customization: A knowledge decision support approach. Artificial Intelligence for Engineering Design, Analysis and Manufacturing, v. 18 , p. $87-109,2004$. http://dx.doi.org/10.1017/ S0890060404040077

ZHANG, X.; CHEN, R. Forecast-driven or customer-orderdriven? An empirical analysis of the Chinese automotive industry. International Journal of Operations \& Production Management, v. 26, n. 6, p. 668-688, 2006. http://dx.doi.org/10.1108/01443570610666993

ZHANG, W. Y.; TOR, S, Y.; BRITTON, G. A. Managing modularity in product family design with functional modeling. International Journal of Advanced Manufacturing Technology, v. 30, p. 579-588, 2005. http://dx.doi.org/10.1007/s00170-005-0112-z

\title{
Definition of critical characteristics in mass customization service implementation
}

\begin{abstract}
This study defines mass customization (MC) characteristics to be prioritized in the design of mass customized services. We propose a classification of mass customization characteristics in the services customization area based on MC enablers and service enablers. These enablers are related using the house of quality matrix from QFD (quality function deployment) and are rewritten after gathering importance inputs from managers familiar with the service under consideration, using a reverse QFD procedure. Using this method, it is possible to determine the main characteristics to be considered in a mass customized service. Two case studies are presented to exemplify the proposed methodological steps, one in an electricity distribution company and another in a private university.
\end{abstract}

\section{Keywords}

Mass customization. Service. QFD. 